\title{
Optimal Scheduling of Electric Vehicles for Ancillary Service Provision with Real Driving Data.
}

Tsagkaroulis, Panagiotis; Thingvad, Andreas; Marinelli, Mattia; Suzuki, Kenta

Published in:

Proceedings of $56<$ sup $>$ th $</$ sup $>$ International Universities Power Engineering Conference

Link to article, DOI:

10.1109/UPEC50034.2021.9548153

Publication date:

2021

Document Version

Peer reviewed version

Link back to DTU Orbit

Citation (APA):

Tsagkaroulis, P., Thingvad, A., Marinelli, M., \& Suzuki, K. (2021). Optimał Scheduling of Electric Vehicles for Ancillary Service Provision with Real Driving Data. In Proceedings of 56 International Universities Power Engineering Conference IEEE. https://doi.org/10.1109/UPEC50034.2021.9548153

\section{General rights}

Copyright and moral rights for the publications made accessible in the public portal are retained by the authors and/or other copyright owners and it is a condition of accessing publications that users recognise and abide by the legal requirements associated with these rights.

- Users may download and print one copy of any publication from the public portal for the purpose of private study or research.

- You may not further distribute the material or use it for any profit-making activity or commercial gain

- You may freely distribute the URL identifying the publication in the public portal 


\section{Optimal Scheduling of Electric Vehicles for Ancillary Service Provision with Real Driving Data}

\author{
Panagiotis Tsagkaroulis \\ Nordic RSC \\ Energinet \\ Copenhagen, Denmark \\ pts@energinet.dk
}

\author{
Andreas Thingvad and Mattia Marinelli \\ Department of Electrical Engineering \\ Technical University of Denmark \\ Roskilde, Denmark \\ \{athing,matm\}@elektro.dtu.dk
}

\author{
Kenta Suzuki \\ Nissan Research Center \\ Nissan Motor Co., Ltd. \\ Yokosuka, Kanagawa, Japan \\ ke-suzuki@mail.nissan.co.jp
}

\begin{abstract}
Electric Vehicles (EV)s can with the right charger and aggregated in large numbers be considered as a large storage unit. If the chargers have bidirectional power converters, EVs connected to the grid, could provide Vehicle-to-Grid (V2G) ancillary services. The annual earnings from delivering frequency-controlled normal operation reserve in Denmark is calculated, based on individual user profiles. The individual earnings are strongly dependant on the driving time, distance and parking time at different locations which determine the availability of each $\mathrm{EV}$ to provide ancillary services. The specific user behaviour is based on a set of telematics data acquired from 7,163 Nissan LEAFs in the United States, with information about every driving and charging sessions during one year. The profit of the individual $\mathrm{EV}$, spreads from 51 to $1654 € /$ year. A data set of one year of system frequency measurements from the Nordic grid is used to calculate the impact of the service provision on the State of charge (SOC).
\end{abstract}

Index Terms-Ancillary service provision, Frequency Control, Electric Vehicles, Vehicle to Grid

\section{INTRODUCTION}

The global number of electric vehicles (EV)s has grown exponentially, since 2010 and reached a global level of 2.1 million EV sales in 2019, and a total stock of 7.2 million [1]. In Denmark, the number of EVs has similarly increased, reaching a total of more than $15,000(0.7 \%$ of the total car fleet) in 2020 [2]. The electrification of the transportation sector will bring both challenges and opportunities to the power system, in terms of additional grid loading, such as simultaneous charging when EV owners return home from their last trip [3], but also as to make the EV an active asset in supporting a stable, economic power system based on renewable energy [4].

The relative significance of the charging control to the revenue from providing frequency regulation, has been explored in [5], and showed that by widening the range of the departure battery level, could increase the revenue. Frequencycontrolled normal operation reserve (FCR-N) is an automatic regulation supplied by production or consumption units, in response to grid frequency deviations. FCR-N is activated for all system frequency deviations within a range of $\pm 100 \mathrm{mHz}$. In conjunction with a rapid frequency change to $49.9 / 50.1 \mathrm{~Hz}$, the power response should be fully implemented within 150 seconds [6]. The service can only be provided by a Balance Responsible Party (BRP) with a minimum bid size of $0.3 \mathrm{MW}$ with the same power for up- and down regulation. All accepted bids, receive an availability payment according to the 'pay-asbid' mechanism [7].

The revenue from FCR-N depends on many parameters such as the capacity of the battery, the frequency energy content but also the electricity prices [8], the plug-in time [9] and the availability to provide FCR-N. By analyzing the individual profiles of EV users and using individual energy deadlines, an additional time for service provision can be allocated, resulting in higher yearly revenue [4].

The rest of the paper is structured as follows: Section II includes the methodology. First the analysis of the provided driving data set, from Nissan [12], is made. In continuation, a description of the system frequency in Denmark follows and afterwards, the energy model of the battery is formulated. The aim of this study is to evaluate the yearly revenue and profit for each EV owner for delivering FCR-N in Denmark. In Section III the maximum potential revenue is found with the proposed model that calculates the optimal the charging and service provision schedule. To that end, Section IV demonstrates the results and Section $\mathrm{V}$ the conclusion of this paper.

\section{METHODOLOGY}

The following subsection presents the principal findings of the current investigation, in the driving data gathered from EVs in the US. The purpose is to explore the resemblance between the driving behavior in US compared to Denmark and validate the relevance of the US data set in this study.

\section{A. Analysis of Driving and Charging Behavior}

The anonymized data from Nissan, consisted of 7,163 Nissan $24 \mathrm{kWh}$ LEAFs, show the daily behaviour of each EV individually during 2015 - 2016. Each EV profile vector consists of data for a whole year with a 15-minute time resolution, which designates whether the EV is on a trip or parked at the household, the workplace or some other location, along with the driving distance and the charging requirements. The driving distance of each trip is expressed in $\mathrm{km}$, while the charging pattern, consists of power measurements expressed in $\mathrm{kWh}$ for the time periods that the EV is parked throughout the day. The locations were obtained based on the GPS telematics data of each EV. Knowing when the EV is in motion, charging 
or parked a different set of useful patterns can be derived. Fig. 1 is a representation of the distribution of the average driving distance per day for all the vehicles. The average distance is slightly higher compared to Denmark, where the average driving distance is $45 \mathrm{~km}$ /day for passenger cars [10]. The national Danish Travel Survey is based on conventional vehicles in Denmark but it is found that the driving distances are similar [9]. It is therefore assumed that EVs in Denmark will have a similar driving behaviour.

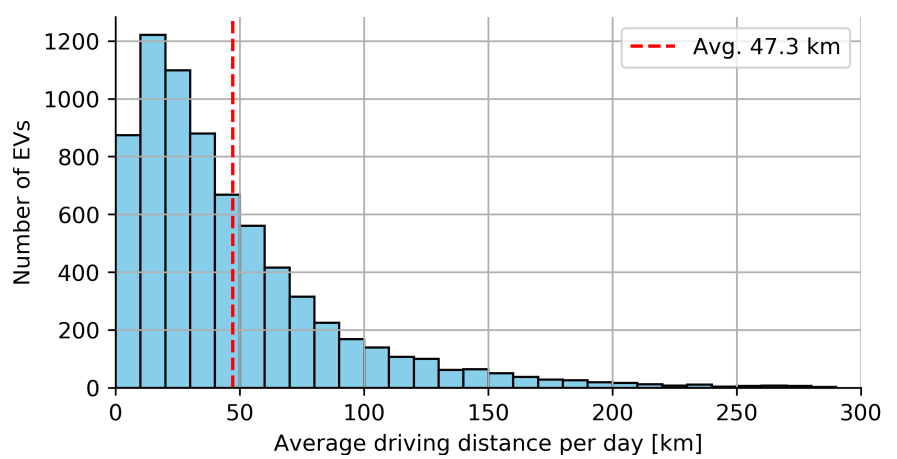

Fig. 1. Distribution of average driving distance per day.

The average yearly driving time is 228 hours/year in U.S, giving an average utilization of $2.6 \%$, which is comparable to driving time in conventional cars in Denmark [10]. The driving distance of each $\mathrm{EV}$ is used to calculate its energy requirements. The driving destination of each trip, determine the availability of each vehicle. If an EV is being driven, it is not available for grid services, thus the fraction of the cars that are driving at the same time determines the number of cars it would require to cover the national demand. Additionally it is assumed that V2G chargers only are installed at the household which limits the locations from which the EV can deliver FCR-N.

\section{B. System Frequency in Denmark}

The frequency of the Nordic power system has been analysed to determine the effect on the SOC of the EVs delivering FCR-N. The data set consists of frequency measurements every $10 s$ during 2016. For a frequency value $f_{t}$ at time $\mathbf{t}$, the normalized response $y_{t}$ of the service provided is calculated as:

$$
y_{t}=\frac{\left(f_{t}-50\right)}{0.1}, \text { if } 49.9 \mathrm{~Hz} \leq f_{t} \leq 50.1 \mathrm{~Hz}
$$

The power required is found by multiplying the normalized response with the contracted power capacity at each hour. $P^{\text {cap }}$ is constant over the hour.

$$
P_{t}=P^{\text {cap }} \cdot y_{t}+P^{\text {base }}
$$

If the frequency on average is above or below $50 \mathrm{~Hz}$, there will be an energy exchange with the grid. This energy content or bias $\mathrm{E}^{\text {bias }}$ is calculated each 15 minutes (same time resolution as the $\mathrm{EV}$ data) as the integral of the normalised response [11].

$$
E_{\tau}^{\text {bias }}=\frac{1}{N_{k}} \sum_{t=N \cdot(\tau-1)+1}^{\tau \cdot N} y_{t} \cdot t_{s}
$$

The sample rate is $t_{s}=10 \mathrm{~s}, N=90$ is the number of samples in each 15 minute period and $N_{k}=3600 \mathrm{~s} / \mathrm{h}$. $\mathrm{E}^{\text {bias }}$ is in p.u. and is multiplied with $\mathrm{P}^{\text {cap }}$ to find the energy content in $\mathrm{kWh}$. The energy content is negative when energy is supplied to the grid and positive when energy is received.

\section{Formulation of Energy Model of the Battery}

The V2G charger has a rated charging/discharging power of $\pm 10 \mathrm{~kW}$, measured at the grid connection on the AC side. The energy consumed from or delivered to the grid $E_{\tau}^{\text {grid }}$ at period ' $\tau$ ', on the AC side is calculated as the sum of the allocated charging power and the energy content scaled with the FCR-N capacity:

$$
E_{\tau}^{\text {grid }}=E_{\tau}^{\text {charge }}+P_{\tau}^{\text {cap }} \cdot E_{\tau}^{\text {bias }}
$$

There is a conversion loss associated with charging and discharging, which is found by assuming a fixed efficiency of $\eta=0.9$ in both directions [15]. When the EV is charging it receives less power than it is received from the grid and when it is discharging the power extracted from the battery is higher compared to the power received from the grid. The following expression shows the calculation of the energy received each 15 minutes from the battery $E^{\text {bat }}$.

$$
E_{\tau}^{\text {bat }}=\left\{\begin{array}{lll}
E_{\tau}^{\text {grid }} \cdot \eta & \text { if } & E_{\tau}^{\text {grid }}>0 \\
E_{\tau}^{\text {grid }} \cdot \frac{1}{\eta} & \text { if } & E_{\tau}^{\text {grid }}<0
\end{array}\right.
$$

Fig. 2 shows the SOC of the battery during a time span of three days, for five real EV profiles, which have been simulated with a battery capacity of $40 \mathrm{kWh}$. The SOC of the battery is affected by the driving consumption during a trip and by the energy content of the frequency grid while connected and delivering FCR-N. The calculation is made, taking the conversion losses of the power converter into consideration. Due to the high variance of $E^{\text {bias }}$ and the effect of the conversion losses. For an EV that delivers FCR-N for many hours consecutively, without charging, the high variance will cause violation of the SOC limits. As in can be seen, the SOC ranges outside of the physical constraints (20-90\% SOC). In a real situation, the violation of the limits would have caused the depletion or saturation of the EV's battery and a failed delivery of FCR-N. To deal with the high variance, an allocation of power for correction of the SOC is essential.

A correction strategy of allocating $P^{\text {cap }}= \pm 7 \mathrm{~kW}$ for FCR$\mathrm{N}$ and reserving $3 \mathrm{~kW}$ for changing the scheduled baseline, when the frequency leaves a specified dead band, is used, in order to keep the SOC within the limits of $20-90 \%$. Value of $P^{\text {base }}=3 \mathrm{~kW}$ was chosen, as it is the minimum amount of power for correcting the SOC while delivering FCR-N that does not result in violation of the SOC limits [9]. The $P^{\text {base }}$ is 


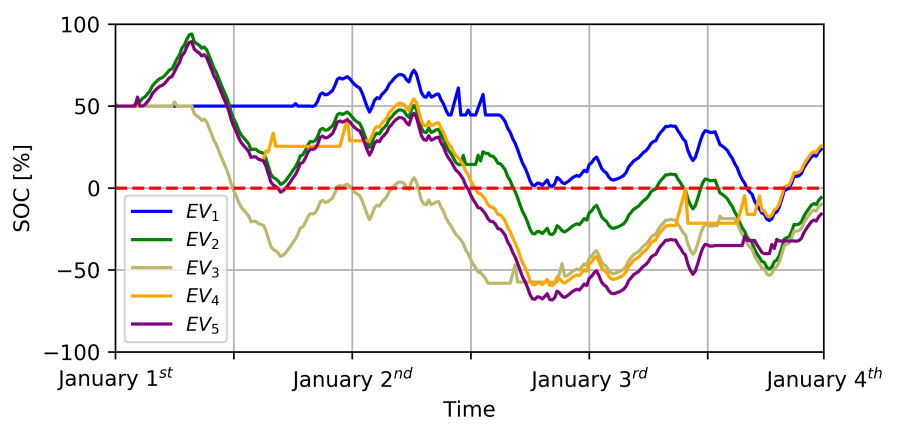

Fig. 2. Simulation result of five Nissan LEAFs, while providing FCR-N, charging and driving for three days. The simulation assumes $40 \mathrm{kWh}$ capacity.

applied when the accumulated energy balance of the battery $\Delta E$ is above $10 \mathrm{kWh}$ or below $-10 \mathrm{kWh}[12]$.

Note, that the time frame is in quarters of an hour, thus the $\mathrm{P}^{\text {base }}$ applied every 15 minutes. The updated $\mathrm{E}_{\tau}^{\text {grid }}$ is calculated based on:

$$
E_{\tau}^{\text {grid }}=E_{\tau}^{\text {charge }}+P_{\tau}^{\text {cap }} \cdot E_{\tau}^{\text {bias }}+E_{\tau}^{\text {base }}
$$

\section{FCR-N capacity payment}

It is considered that all FCR-N bids are accepted and will receive the weighted average capacity payment for that hour. The capacity payment for FCR-N $\left(\mathrm{c}_{\tau}\right)$ in Denmark is compensated with the price per MW per hour when the service is made available. The service is only sold if the EV is available at home during the full hour and the price is the same for the whole hour, but the final yearly revenue is calculated for each 15-minute.

$$
\text { Revenue }=\sum_{\tau=1}^{365 \cdot 24 \cdot 4} c_{\tau} \cdot P_{\tau}^{\text {cap }}
$$

Fig. 3 shows the hourly average values of the FCR-N prices for five consecutive years. In general the average capacity payment is significantly higher during the night compared to the morning hours. The prices for every day during 2019 are also presented as grey lines in Fig. 3. The following simulations are calculated with the FCR-N prices of the specific hours during 2019. The high variation during the day is strongly correlated with the supply at each hour, and the yearly average price is $30.34 € / \mathrm{MWh}$.

The yearly cost derived from the energy losses, is calculated with the hourly spot prices plus the average industrial tariff $\gamma^{\text {tariff }}$, where the $D S O_{\text {tariff }}$ is equal to $0.0376 € / \mathrm{kWh}$ [13].

$$
\operatorname{Cost}_{V 2 G}=\sum_{\tau=0}^{365 \cdot 24 \cdot 4}\left(P_{\tau}^{\mathrm{cap}} \cdot E_{\tau}^{\mathrm{bias}}+E_{\tau}^{\mathrm{base}}\right) \cdot\left(\lambda_{\tau}+\gamma^{\mathrm{tariff}}\right)
$$

\section{OPTIMIZATION OF CHARGING AND SERVICE SCHEDULE}

In this section a method for calculating the best case earnings for every car in an optimisation problem with full

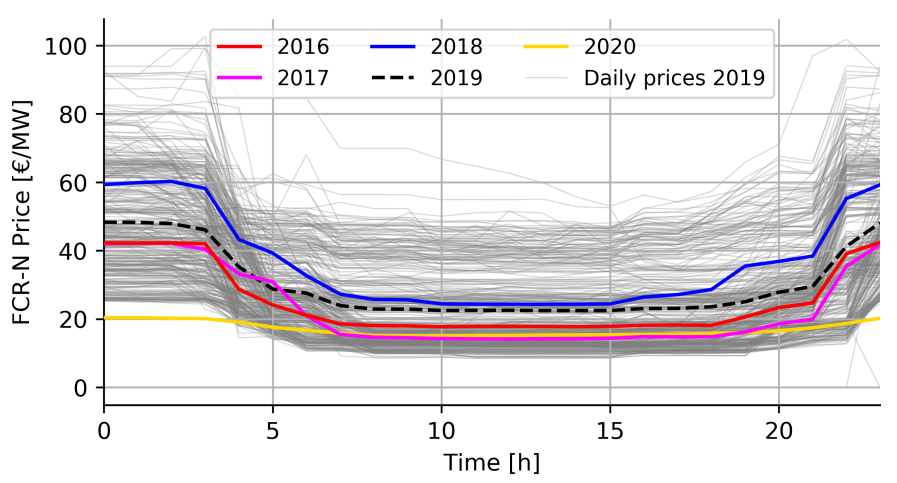

Fig. 3. The average value of FCR-N price per hour, for 2016, 2017, 2018, 2019, 2020 and the daily prices during 2019.

knowledge of future driving consumption and future energy content of the frequency is presented.

\section{A. Problem description}

The main objective of this optimization proposal is to maximize the profit of each EV by minimizing its costs, while respecting a set of constraints related to power limit of the V2G charger while charging, discharging and providing FCR$\mathrm{N}$ (10), (11). It is assumed that the EV has access to the V2G charger while it is located at the owner's home (12). Constraint (13) avoids charging and discharging at the same time, where $S_{\mathrm{i}, \mathrm{j}}^{c}, S_{\mathrm{i}, \mathrm{j}}^{d}$ can be either 1 or 0 . To prevent the EV from providing FCR-N (14), charging (15) or discharging (16) while it is being driven, another set of constraints are considered, where $S_{\mathrm{i}, \mathrm{j}}^{c a p}$ can be either 1 or 0 . The SOC level is calculated through the cumulated charging, discharging, FCR-N energy and driving energy consumption $\left(E^{\text {drive }}\right)(18)$. Lastly, the SOC limits are set between $20 \%$ and $90 \%$ (19). The prices for the capacity payment and the spot prices are provided for each hour during 2019. For the examined case, the average tariffs $\gamma^{\text {tariff }}$ will be added on top of the price for charging and discharging. The value of the $P^{\text {cap }} \mathrm{V} 2 \mathrm{G}$ charger is $\pm 10 \mathrm{~kW}$ and the value of the battery is $\mathrm{Q}=40 \mathrm{kWh}$. A constant charging and discharging efficiency, $\eta=0.9$ is considered for all loading levels. The time step $(\Delta \mathrm{i})$ is one hour since the prices are updated hourly. During this hour the charging, discharging or the frequency service procurement is considered constant. Moreover, the time horizon, I, of the optimization is 8760 hours. Since $\Delta_{i}$ is one hour, the power for charging $\left(P^{\mathrm{c}}\right)$ is constant during that hour and the same applies for discharging $\left(P^{\mathrm{d}}\right)$. The spot price $\left(\lambda_{i}\right)$ for the market at Nord Pool reflects the cost of energy in $€ / \mathrm{kWh}$ while the capacity payment $\left(c_{i}\right)$ for FCR-N is in $€ / \mathrm{kW}$ per hour.

\section{B. Formulation}

The objective function of the optimization problem contains the cost of the energy for charging and the revenue for discharging and FCR-N payment. The sign of the revenue sources are negative and for this reason the objective function should be minimised, which is subject to a set of equality and inequality constraints, described in subsection III-A. The 
15-min values for $E^{\text {drive }}$ and $E^{\text {bias }}$ are aggregated to hourly values. The investment cost for the charger is not taken into consideration.

$$
\min \sum_{i=0}^{I} \sum_{j=0}^{J} \lambda_{i} \cdot P_{i, j}^{\mathrm{c}}-\lambda_{i} \cdot P_{i, j}^{\mathrm{d}}-c_{i} \cdot P_{i, j}^{\mathrm{cap}}
$$

s.t.

$$
\begin{gathered}
P_{i, j}^{\mathrm{cap}}+P_{i, j}^{\mathrm{c}}+P_{i, j}^{\mathrm{d}} \leq P_{\max } \quad \forall i \in I, j \in J \\
P_{i, j}^{c}, P_{i, j}^{\mathrm{d}}, P_{i, j}^{\mathrm{cap}} \geq 0 \quad \forall i \in I, j \in J \\
P_{i, j}^{\mathrm{cap}} \cdot \sigma_{i, j}=0, \quad P_{i, j}^{\mathrm{d}} \cdot \sigma_{i, j}=0 \quad \forall i \in I, j \in J \\
S_{i, j}^{\mathrm{c}}+S_{i, j}^{\mathrm{d}} \leq 1 \quad \forall i \in I, j \in J \\
S_{i, j}^{\mathrm{cap}} \cdot E_{i, j}^{\mathrm{drive}}=0, \\
S_{i, j}^{\mathrm{cap}} \cdot P_{\min } \leq S_{i, j}^{\mathrm{cap}} \cdot P_{i, j}^{\mathrm{cap}} \leq S_{i, j}^{\mathrm{cap}} \cdot P_{\max } \quad \forall i \in I, j \in J \\
S_{i, j}^{\mathrm{c}} \cdot E_{i, j}^{\mathrm{drive}}=0, \quad \forall \\
S_{i, j}^{\mathrm{c}} \cdot P_{\min } \leq S_{i, j}^{c} \cdot P_{i, j}^{\mathrm{c}} \leq S_{i, j}^{\mathrm{c}} \cdot P_{\max } \quad \forall i \in I, j \in J \\
S_{i, j}^{\mathrm{d}} \cdot E_{i, j}^{\mathrm{drive}}=0, \quad \forall i \in I, j \in J \\
S_{i, j}^{\mathrm{d}} \cdot P_{\min } \leq S_{i, j}^{\mathrm{d}} \cdot P_{i, j}^{\mathrm{d}} \leq S_{i, j}^{\mathrm{d}} \cdot P_{\max } \quad \forall i \in S_{i, j}^{\mathrm{c}}, S_{i, j}^{\mathrm{cap}} \in\{0,1\} \quad \forall i \in I, j \in J \\
\sigma_{i, j}, S_{i, j}^{\mathrm{c}}, S_{i, j}^{\mathrm{d}}=S_{i, j}+\left[E_{i, j}^{\mathrm{drive}}\right. \\
S O C_{i+1, j} \\
\left.+P_{i, j}^{\mathrm{cap}} \cdot E_{i, j}^{\mathrm{bias}}+P_{i, j}^{\mathrm{c}} \cdot \eta-P_{i, j}^{\mathrm{d}} \cdot \frac{1}{\eta}\right] \frac{1}{Q} \quad \forall i \in I, j \in J \\
\frac{S O C_{i, j} \leq S O C_{i, j}=0 \ldots J}{S O C_{i, j}} \quad \forall i \in I, j \in J
\end{gathered}
$$

Where, ' $\sigma$ ' is vector that indicates the availability of the $\mathrm{EV}$ for FCR-N provision. It equals 1 when the EV is parked at the owner's home and 0 when the EV is on a trip or parked somewhere else.

\section{RESULTS}

\section{A. Driving and Charging Behaviour}

The driving behavior is different throughout work days (Monday- Friday) and weekend days (Saturday-Sunday). Fig. 4 shows the fraction of the 7,163 EVs that are driving, at the same time on a workday (Monday-Friday) and Fig. 5 for a weekend day (Saturday-Sunday), based on one year of data. New Year's day, Christmas day, Thanksgiving's and the day after, are included in the weekend days as they are public holidays.

Fig. 6 shows the fraction of the 7,163 EVs that are charging, at the same time on a work day and Fig. 7 for a weekend day. From the graphs, it can be observed that the EV driving and charging pattern are different throughout the week days and weekends. The share of EVs that are driving on weekends is noticeably lower, especially during morning and evening hours, without any remarkable peaks. Additionally, the fraction of EVs that are charging during evening hours in the weekdays is higher compared to weekends. Despite the fact that the data is from EVs with $24 \mathrm{kWh}$ battery capacity, the charging peak is considerably lower for all the EVs charging

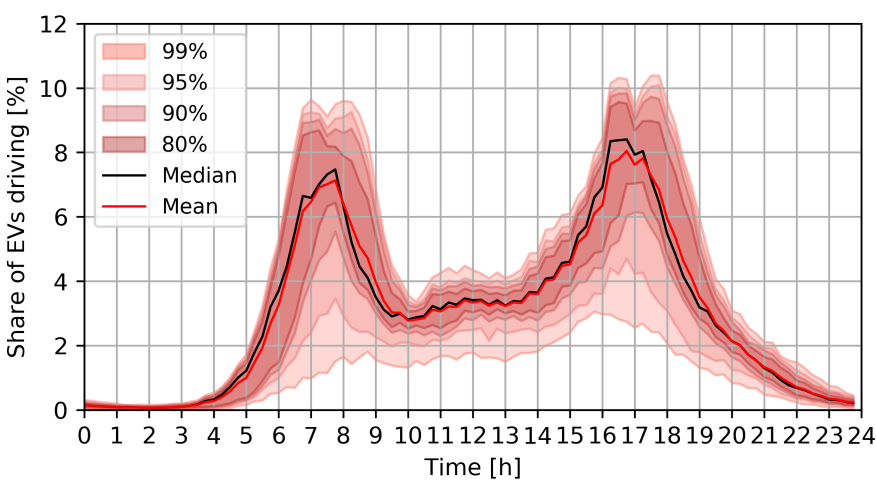

Fig. 4. Fraction of 7,163 EVs that are driving with different confidence intervals during 257 work days (Monday-Friday).

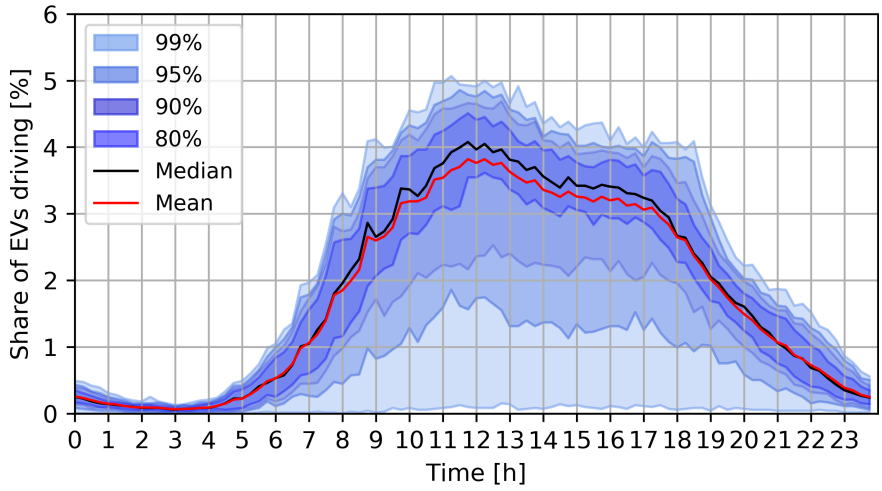

Fig. 5. Fraction of 7,163 EVs that are driving with different confidence intervals, during 108 weekend days (Saturday-Sunday).

concurrently and that is a sign that the owners do not charge their vehicle every day, as long as they don't have to drive a very long distance the next day [14]. Hence, it is reasonable to conclude that this is a good sign in regard to grid congestion.

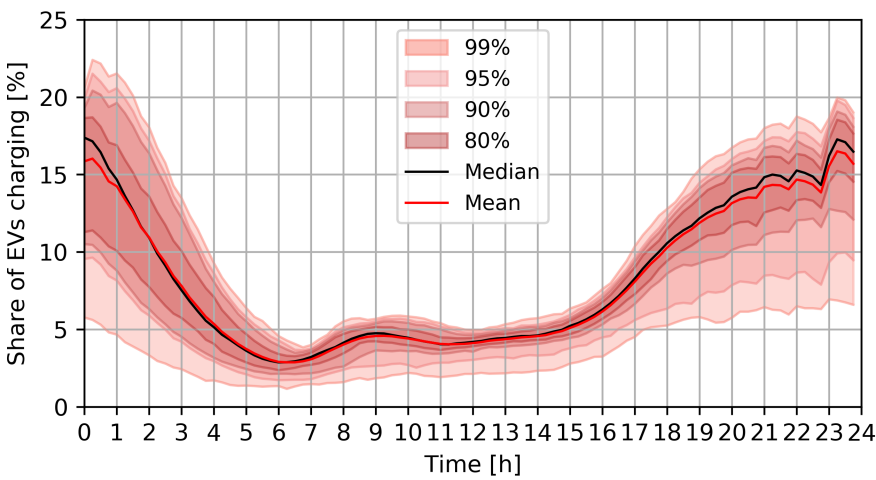

Fig. 6. Fraction of EVs that are charging on work days with different confidence intervals.

\section{B. Heuristic Model}

The fraction of all the EVs that are parked at different location described as 'Home', 'Work' or 'Other' is illustrated in Fig. 8, for a weighted average day including both weekdays and weekends in a year. The share of EVs at home drops below $50 \%$ in the middle of the day and rises up steadily from the 


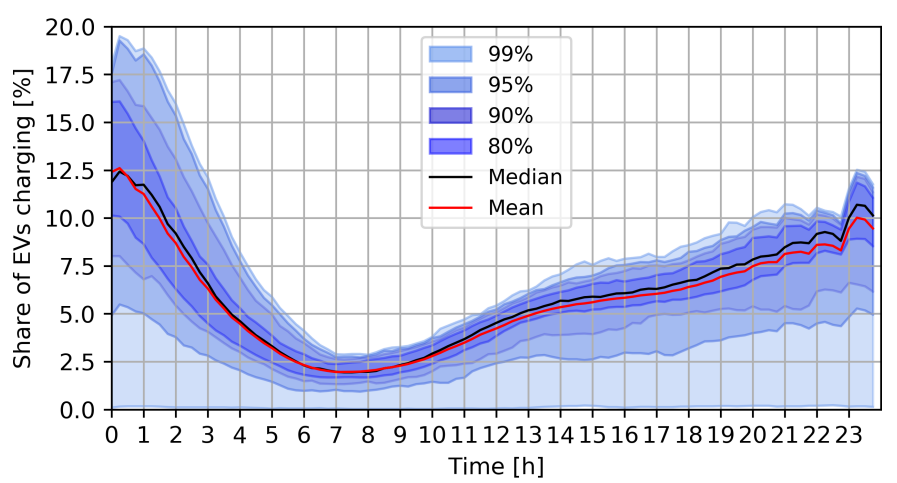

Fig. 7. Fraction of EVs that are charging on weekends, with different confidence intervals.

afternoon, while the number of vehicles at work rises during the day and steadily declines during night, since the people are at work, during these hours. It is also worth mentioning that although the availability of vehicles at home is more than $70 \%$ during morning and night hours, only a small percentage is charging as it is shown in Fig.6 and 7. Additionally, a significant fraction of EVs, which is approximately $40 \%$ during the midday, is located in some other unknown location. The remainder EVs needed to reach $100 \%$ are being driven, as it is demonstrated in Fig.4.

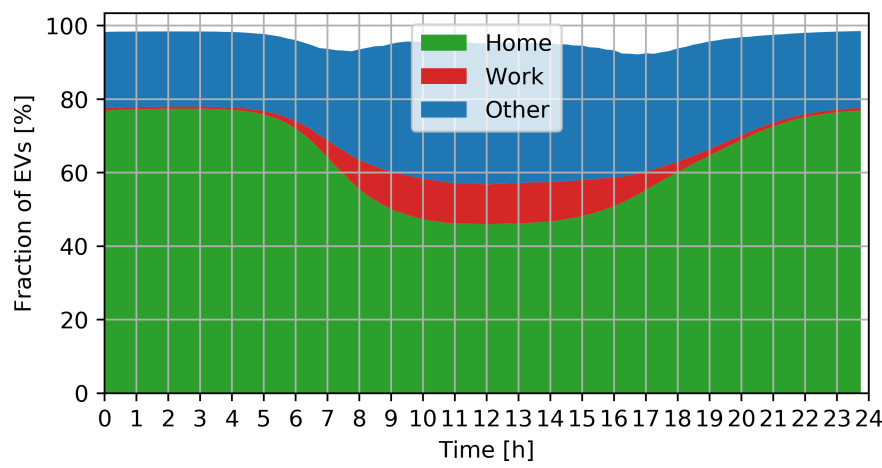

Fig. 8. Fraction of EVs when located at home, work or other location, both work days and weekends in a year.

Fig. 9 shows the percentage of all the vehicles that are available with a certain confidence to provide services to the grid, during weekdays, when they are located at home. The fact that a large fraction of the EVs are parked at home during the working hours shows that even by just having V2G chargers installed at the household it would be possible to deliver FCR$\mathrm{N}$ from EVs in these hours. Energinet continuously procures $23 \mathrm{MW}$ of FCR-N for Eastern Denmark (DK-2). If a group of EVs had V2G chargers at home and could deliver FCR-N with $P^{\text {cap }}=7 \mathrm{~kW}$ with a minimum availability of $30 \%$, it means that the full regulation requirement could be supplied by $10,952 \mathrm{EVs}$.

The SOC of each of the 7,163 EVs is calculated as they were delivering FCR-N every hour that they were parked at home and not charging. The SOC is affected by the driving consumption, charging, conversion losses and energy content.

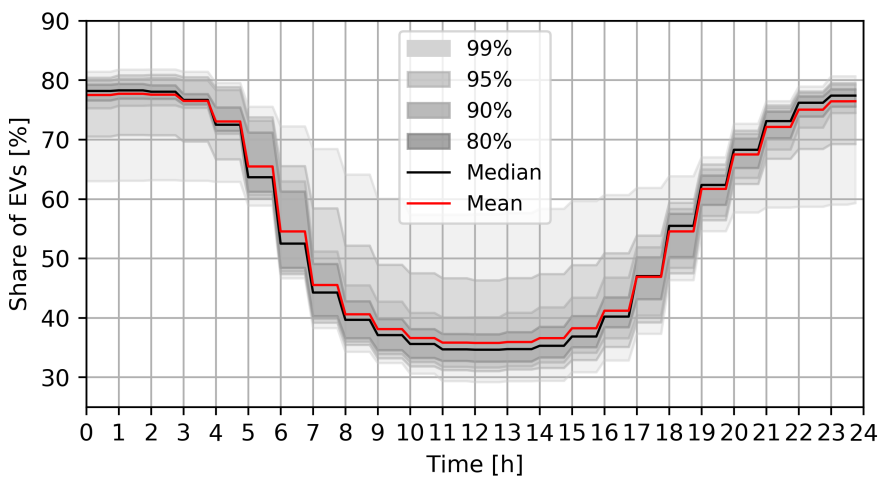

Fig. 9. Share of 7,163 EVs that are available at home to provide FCR-N with confidence intervals, work days.

In Fig. 10 is depicted the resulted SOC of the EV that each 15 minutes during the year has the maximum and minimum SOC as well as the $1^{\text {st }}$ and 99 th percentile. Each point might be a different EV, since the results were sorted from the lowest to the highest value each 15 minutes. The energy ranges between $20-90 \%$ corresponding to [8 - 36] kWh for a $40 \mathrm{kWh}$ battery, with a few outliers. The control strategy, tries to maintain it within $\pm 10 \mathrm{kWh}$ but if the regulation period is followed by a long trip, it will charge to a higher level to accommodate that consumption. This shows that it is a realistic charging and regulation strategy for an EV with that battery size, however the variance is high.

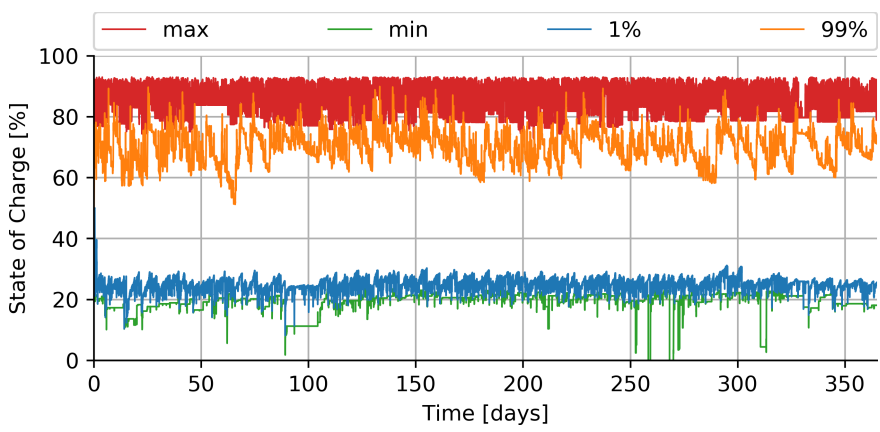

Fig. 10. State of Charge of the battery for all 7,163 EVs, for every day of the year.

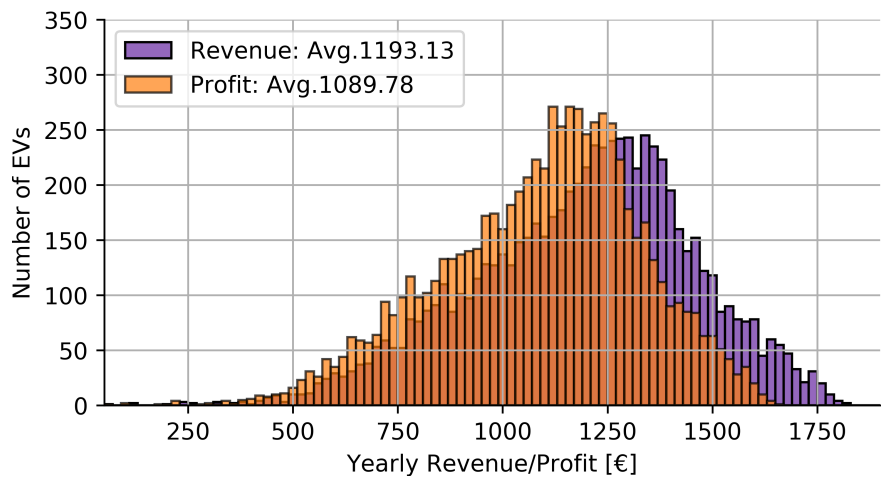

Fig. 11. Distribution of the yearly revenue and profit when EV is located at home, for $7,163 \mathrm{EVs}$ 
The yearly capacity payment for the different EVs is calculated for the case where FCR-N is delivered with $7 \mathrm{~kW}$ all full hours that the EV is at the household, and shown in Fig. 11 . The average yearly revenue of all the EVs is $1193 €$ /year. After subtracting the electricity cost for conversion losses, the profit is $1090 €$ /year. The distribution of the revenue ranges between 56 - $1824 €$ /year, depending on the availability.

\section{Optimization results}

The yearly revenue from a random fraction of $1000 \mathrm{EVs}$ are shown in Fig. 12. The heuristic method resulted in earnings from FCR-N spread between 367 - $1782 €$ /year, with an average value of $1194 €$ /year. By optimizing the charging and service provision schedule of the same EVs it was possible to increase the average yearly revenue by $334 €$ to $1528 €$. The individual EVs are then spread between 475 - $2278 €$ e/year For the heuristic method $P^{\text {cap }}$ was fixed at $\pm 7 \mathrm{~kW}$ due to the $3 \mathrm{~kW}$ allocated for $P^{\text {base }}$. The optimisation on the other hand chose $P^{\text {cap }}$ for every hour to avoid violating the hard SOC constraints.

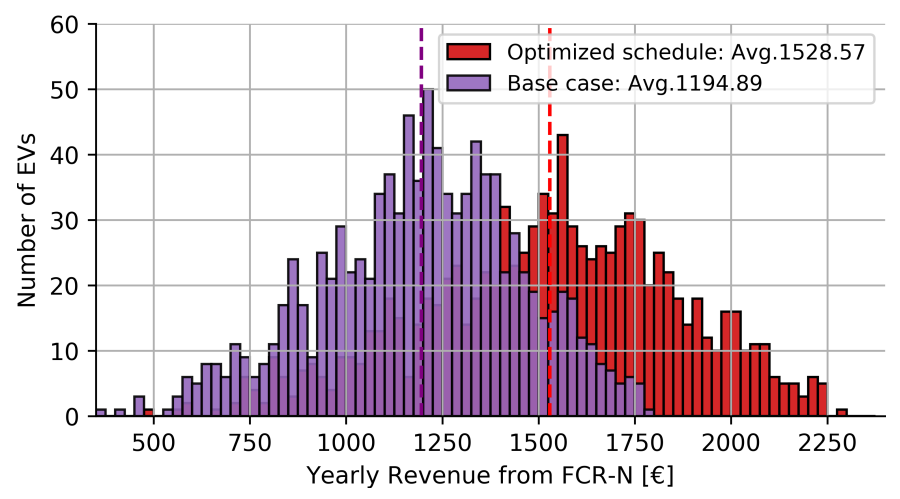

Fig. 12. Comparison of the distributed yearly revenue, when the charging and service provision schedule is optimized and the base case, for 1000 EVs.

The EV is delivering FCR-N during the hours it is at home, and discharges when the spot price is high and charges when the spot price is low, considering that the model is economically feasible. The SOC of the battery varies a lot, as an effect of the energy content. However, it remains within the chosen limits, [20\% - 90\%]. In the simulated scenario the driving consumption but also the time of the upcoming trip are known. However, in a real life scenario, the EV owner can leave at any time. Considering this, the EV's battery might not have enough energy to accommodate the needs of an upcoming long trip. Therefore, it is of great importance to have a large set of historic driving data to rely on, when estimating the precision of the plug-in and plug-out time.

The income of the most profitable EVs is highly dependant on the availability hours to provide FCR-N, when they are parked at home, compared to the least profitable. Therefore the availability hours for the highest revenue EV are 8572, compared to only 296 hours, for the EV with the least.

\section{CONCLUSION}

In this study the EV driving and charging behaviour of a large EV fleet is analysed, by performing a statistical analysis on the examined data set. Based on the driving and charging data the possible earnings from FCR-N provision of the individual car is investigated. This is calculated with a heuristic method which serves as a benchmark for the presented optimisation model. The optimization model is proposed for scheduling both FCR-N provision and charging, to calculate the income of each EV owners, assuming full knowledge on the prices in the day-ahead market. The model assumes full knowledge of the future system frequency behaviour and charging and driving patterns of the EV owners so it describes the best case earnings. The optimization results show that there is a high margin in increasing each EV's individual revenue and it is strongly dependant upon the availability of the EV.

\section{ACKNOWLEDGEMENT}

The work in this paper has been supported by the research projects ACES (EUDP grant nr: EUDP17-I-12499) and ACDC (EUDP grant nr. 64019-0541). Website: www.acesbornholm.eu, www.acdc-bornholm.eu

\section{REFERENCES}

[1] International Energy Agency, 'IEA (2020), Global EV Outlook 2020 IEA, Paris, https://www.iea.org/reports/global-ev-outlook-2020'

[2] I. Wagner, Stock of electric cars in Denmark 2010-2020, Statista 2021, https://www.statista.com.

[3] EL. Karfopoulos , ND. Hatziargyriou (2013) A multi-agent system for controlled charging of a large population of electric vehicles. IEEE Trans Power Syst 28(2):1196-1204.

[4] P. B. Andersen, T. Sousa, A. Thingvad, L. S. Berthou, M. Kulahci,(2018). Added value of individual flexibility profiles of electric vehicle users for ancillary services. In Proceedings of IEEE International Conference on Communications, Control, and Computing Technologies for Smart Grids IEEE, pp 1-6.

[5] Han, Sekyung et al. "Development of an Optimal Vehicle-to-Grid Aggregator for Frequency Regulation." IEEE Transactions on Smart Grid 1 (2010): 65-72.

[6] Explanatory document for the Nordic synchronous area proposal for additional properties of FCR in accordance with Article 154(2) of the Commission Regulation (EU) 2017/1485 of 2 August 2017 establishing a guideline on electricity transmission system operation.

[7] Energinet.dk, Ancillary services to be delivered in Denmark tender conditions, 2019

[8] L. Calearo, M. Marinelli, (2020). Profitability of frequency regulation by electric vehicles in Denmark and Japan Considering Battery Degradation Costs. World Electric Vehicle Journal, 11(3), [48].

[9] A. Thingvad, C. Ziras, and M. Marinelli, "Economic value of electric vehicle reserve provision in the nordic countries under driving requirements and charger losses," Journal of Energy Storage., 2019.

[10] Liu Zhaoxi, Wu Qiuwei, D2.2. EV charging analysis with high EV penetration in the Nordic region", 2013.

[11] A. Thingvad, C. Ziras, J. Hu, and M. Marinelli, Assessing the energy content of system frequency and electric vehicle charging efficiency for ancillary service provision, 2017.

[12] A. Thingvad, L. Calearo, P. B. Andersen, M. Marinelli, M. Neaimeh, K. Suzuki, K. Murai, (2019). Value of v2g frequency regulation in Great Britain considering real driving data. In Proceedings of 2019 IEEE PES Innovative Smart Grid Technologies Europe [8905679] IEEE.

[13] El-Net Øst A/S, https://elnetoest.dk/priser/

[14] L. Calearo, A. Thingvad, K. Suzuki, M. Marinelli, (2019). Grid Loading due to EV Charging Profiles Based on Pseudo-Real Driving Pattern and User Behaviour. IEEE Transactions on Transportation Electrification, 5(3), 683-694.

[15] A. Thingvad, C. Ziras, M. Marinelli, (2019). Economic value of electric vehicle reserve provision in the Nordic countries under driving requirements and charger losses. Journal of Energy Storage, 21, 826834. 